\title{
National surveillance of pandemic influenza A(H1N1) infection-related admissions to intensive care units during the 2009-10 winter peak in Denmark: two complementary approaches
}

S Gubbels (gub@ssi.dk) ${ }^{1,2}$, A Perner 3, P Valentiner-Branth ${ }^{1}, K_{\text {M }}$ lbak $^{1}$

1. Department of Epidemiology, Statens Serum Institut, Copenhagen, Denmark

2. European Programme for Intervention Epidemiology Training (EPIET), European Centre for Disease Prevention and Control, Stockholm, Sweden

3. Intensive care unit, Rigshospitalet, Copenhagen, Denmark

Citation style for this article:

Gubbels S, Perner A, Valentiner-Branth P, Mølbak K. National surveillance of pandemic influenza A(H1N1) infection-related admissions to intensive care units during the 2009-10 winter peak in Denmark: two complementary approaches. Euro Surveill. 2010;15(49):pii=19743. Available online: http://www.eurosurveillance.org/ ViewArticle.aspx?Articleld $=19743$

Surveillance of 2009 pandemic influenza $A\left(\mathrm{H}_{1} \mathrm{~N}_{1}\right)$ in Denmark was enhanced during the 2009-10 winter season with a system monitoring the burden of the pandemic on intensive care units (ICUs), in order to inform policymakers and detect shortages in ICUs in a timely manner. Between week 46 of 2009 and week 11 of 2010, all 36 relevant Danish ICUs reported in two ways: aggregate data were reported online and case-based data on paper. Cases to be reported were defined as patients admitted to an ICU with laboratory-confirmed 2009 pandemic influenza $A\left(\mathrm{H}_{1} \mathrm{~N}_{1}\right)$ infection or clinically suspected illness after close contact with a laboratory-confirmed case. Aggregate numbers of cases were reported weekly: during weeks 48-51 (the peak), reporting was daily. The case-based reports contained demographic and clinical information. The aggregate surveillance registered 93 new cases, the case-based surveillance 61, of whom 53 were laboratory confirmed. The proportion of beds used for influenza patients did not exceed $4.5 \%$ of the national capacity. Hospitals with cases used a median of $11 \%$ of bed capacity (range: $3-40 \%$ ). Of the patients for whom information was available, 15 of 48 patients developed renal insufficiency, 19 of 50 developed septic shock and 17 of 53 died. The number of patients with pandemic influenza could be managed within the national bed capacity, although the impact on some ICUs was substantial. The combination of both reporting methods (collecting aggregate and case-based data) proved to be useful for monitoring the burden of the pandemic on ICUs.

\section{Introduction}

The first case of 2009 pandemic influenza $A\left(\mathrm{H}_{1} \mathrm{~N}_{1}\right)$ in Denmark was diagnosed on 1 May 2009. The incidence, assessed as the percentage of influenza-like illness (ILI) seen by general practitioners in the national sentinel system, rose in July 2009 and remained stable at around $0.75 \%$ for many months, until it started rising again in the week of 8 November 2009 (week 45) and peaked at $5.03 \%$ in the week of 22 November 2009 (week 47) [1]. Surveillance of ILI seen by the Danish medical on-call service showed a similar pattern [2]. Considering that the age distribution of patients with pandemic influenza as well as the distribution of risk factors differed from those seen in seasonal influenza [3-5], the impact on the healthcare system was also likely to be different from that during a seasonal influenza epidemic. Moreover, as the pandemic vaccine was available in week 45, a vaccination campaign after that would possibly not have been able to prevent many of the severe cases. It was therefore important to monitor severe disease due to the pandemic influenza.

Surveillance systems were enhanced to include hospitalisations and admissions to intensive care units (ICUs), as recommended in the Danish influenza pandemic plan [6]. The surveillance system to monitor the burden on ICUs was created in weeks 45 and 46 of 2009 in cooperation with the ICU of the Copenhagen University Hospital, Rigshospitalet, Denmark. The Danish Society for Anaesthesia and Intensive Care endorsed the system and the National Board of Health recommended that all ICUs in Denmark participated in the reporting. The system was set up to assess the ICU bed capacity used for pandemic influenza patients, and to provide demographic and clinical data as well as risk factors for death in order to estimate the impact of the pandemic on ICUs and contribute to an assessment of the severity of the pandemic and the severity of disease.

\section{Methods}

Clinical notification of patients with pandemic influenza was not mandatory in Denmark. Danish ICUs were, however, requested to report two types of data 
to the Statens Serum Institut: (i) aggregate numbers of pandemic influenza patients by age group and (ii) clinical information for each individual patient. A case that should be reported was defined as a patient admitted to an ICU with laboratory-confirmed pandemic influenza $A\left(\mathrm{H}_{1} \mathrm{~N}_{1}\right)$ infection or a patient whose infection was clinically suspected and had had close contact with a patient with laboratory-confirmed pandemic influenza.

All hospitals with acute care facilities $(n=53)$ in the five hospital regions of Denmark, excluding the Faroe Islands, were invited to take part in the surveillance system. The system started in week 46 of 2009 and was planned to continue until week 20 of 2010 , or until no new cases had been reported by the ICUs for three consecutive weeks, and other surveillance systems, such as the sentinel system, also showed low and stable incidence levels.

\section{Aggregate data}

Starting on 15 November 2009 (week 46), ICUs reported aggregate data once a week on a Monday morning before 12:00. During weeks 48 to 51 inclusive of 2009, they were asked to report on a daily basis. Then the deadline was 09:00 on Mondays to Thursdays; data for Fridays and the weekends were reported on Mondays.

A web-based reporting form was created on the homepage of the Statens Serum Institut. A dedicated contact person in the ICUs reported the number of new cases, as well as the number of cases present in the ICU at 08:00 on the day of reporting. The number of cases was reported by the following age groups: «1 year, 1-4 years, 5-14 years, 15-24 years, 25-64 years, $65-74$ years and $\geq 75$ years.

We entered the data from the web-based form to a master dataset in a Microsoft Access database. Each report in the aggregate system was evaluated and validated. Reports were corrected for double reporting when a case was transferred to another hospital, but this could only be done if the hospitals actively informed us. Similarly, reports were amended or removed when we were informed of errors or when they contained obvious inconsistencies that needed further follow-up. Bed capacity, expressed as a percentage, was calculated as the number of cases present in an ICU divided by the total number of beds available at that moment.

A summary of the data received was disseminated to the ICUs and the National Board of Health once a week and each day during weeks 48-51 of 2009 (the winter peak). The National Board of Health presented these reports in the parliamentary standing committee on health.

\section{Case-based data}

The form used to gather information on each patient included demographic and clinical data, such as underlying medical conditions, co-presenting illnesses, dates of onset of symptoms and admission to ICU and details on treatment. A physician completed this paper form. ICUs were asked to send the completed forms as soon as possible after a patient was admitted and to send any additional information at a later stage if anything was unknown on admission.

A unique patient identifier (the person's number from the Danish Civil Registry System [7]) was reported on the case-based form. The Civil Registration number enabled us to complement the case-based surveillance with data from several registers. From the Danish Civil Registry System we could verify cases who had died as a result of pandemic influenza. A case who died of pandemic influenza after ICU admission was defined as a patient reported in the case-based surveillance who died within 30 days after initial laboratory confirmation of the infection. The Statens Serum Institut laboratory database was used to verify the laboratory confirmation of the patients reported in the case-based surveillance. During the pandemic, laboratories in Denmark were obliged to send samples from patients with ILI to the reference laboratory in Statens Serum Institut, either for initial testing or for confirmation. Vaccination status was verified using the Danish vaccination registry, which was set up in 2009 and was assumed to cover the majority of pandemic vaccine recipients. The vaccination registry also included the reason for vaccination.

Data were analysed using Fisher's exact test for categorical variables with a binary outcome and the MannWhitney test for continuous variables. The level of significance was set at p<0.05.

\section{Results}

We implemented the pandemic surveillance system, both for aggregate and individual data in week 46 of 2009. The system was discontinued after week 11 of 2010 as no new cases had been reported for three consecutive weeks and both sentinel surveillance and oncall monitoring showed low activity for several weeks [2].

Of the 53 hospitals in Denmark with acute care facilities, five had no ICU and 16 were part of a larger group of hospitals that reported for them. As a result 32 hospitals across Denmark were identified for reporting. They reported for 36 ICUs: 32 general ICUs, two paediatric ICUs and two ICUs for neurosurgery.

\section{Aggregate data}

All 36 ICUs took part in the surveillance system, although the level of participation varied: until week 8 of 2010 the number of reporting ICUs varied between 22 and 29 after which the numbers dropped to 15 and 16, in weeks 10 and 11 of 2010, respectively. Late reports usually did not contain any cases. Personal contact with hospitals that had a low response rate confirmed that they had not reported because they had no cases. 
After data cleaning, 355 weekly and 758 daily reports were validated and used for analysis. During the surveillance period 93 new cases were reported. Figure 1 shows the number of new cases by week of admission and the timeliness of reporting. Late reports were usually received within a week after the deadline. Only two cases admitted during the Christmas week were reported two weeks later. Data from the national sentinel surveillance system were added, showing the proportion of patients with ILI among the total number of patients who consulted a general practitioner. The peak of new pandemic influenza cases in ICUs was seen in week 48 of 2009 , one week later than the peak seen in the sentinel data and two weeks after the oncall monitoring peak [2]. The last new case in an ICU was reported in week 8 of 2010 .

The proportion of beds used for pandemic influenza cases did not exceed $4.5 \%$ of the total national ICU bed capacity. Hospitals with cases used a median of $11 \%$ of ICU beds for pandemic influenza patients (range: $3-40 \%)$.

\section{Case-based data}

A total of 74 case-based forms were received from 19 hospitals. These forms contained details of 61 individual patients: for 13 patients we received a second, updated form, either from the same hospital or from another hospital to which the patient had been transferred. Of the 61 reported cases, 53 were laboratory confirmed by polymerase chain reaction (PCR). Four cases tested negative in several PCR tests; for another four, the laboratory results could not be traced. Only the 53 laboratory-confirmed cases were used for analysis.

The number of laboratory-confirmed cases from the case-based surveillance is shown in Figure 2 by week of admission, as well as data from the sentinel system. Unlike the epidemic curve of the aggregate data, the peak of the case-based data coincided with the peak of the sentinel data and was one week after the on-call monitoring peak [2].

\section{Demographic data}

Of the 53 laboratory-confirmed cases, 31 were male and 22 were female. The median age was 47 years (range: $3-80$ years). Figure 3 shows the age- and sexspecific incidence. The median age among men was 50 years (range: $3-75$ years) and among women 45 years (range: $5-80$ years; Mann-Whitney test $\mathrm{p}=0.96$ ).

\section{Medical history}

Details on medical history were complete for most cases, but for a few patients some details were missing. The presence or absence of an underlying medical condition was reported for 52 of the 53 laboratory-confirmed cases: 11 had no pre-existing underlying medical condition, while 41 had at least one. Table 1 shows the underlying conditions for cases under 15 years of age and those aged 15 years and older. The presence of specified underlying illnesses varied between 9 of 47

\section{FIGURE 1}

Weekly aggregate data: reported new 2009 pandemic influenza $A(\mathrm{H} 1 \mathrm{~N} 1)$ cases by week of admission $(n=93)$ by timeliness of reporting and data from the national sentinel system, Denmark, week 40 of 2009 to week 11 of 2010

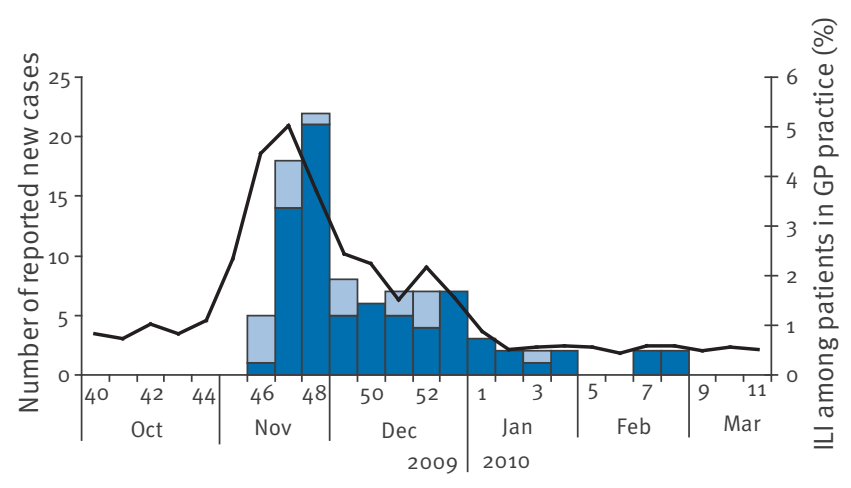

Week and month of admission

$$
\begin{aligned}
& \text { Cases reported after deadline } \\
& \text { Cases reported on time } \\
& ـ \text { Percentage of ILI among patients in GP practice (sentinel data) }
\end{aligned}
$$

GP: general practioner; ILI: influenza-like illness.

\section{FIGURE 2}

Case-based data: laboratory-confirmed 2009 pandemic influenza $A(H 1 N 1)$ cases by week of admission $(n=53)$ and data from the national sentinel system, Denmark, week 40 of 2009 to week 11 of 2010

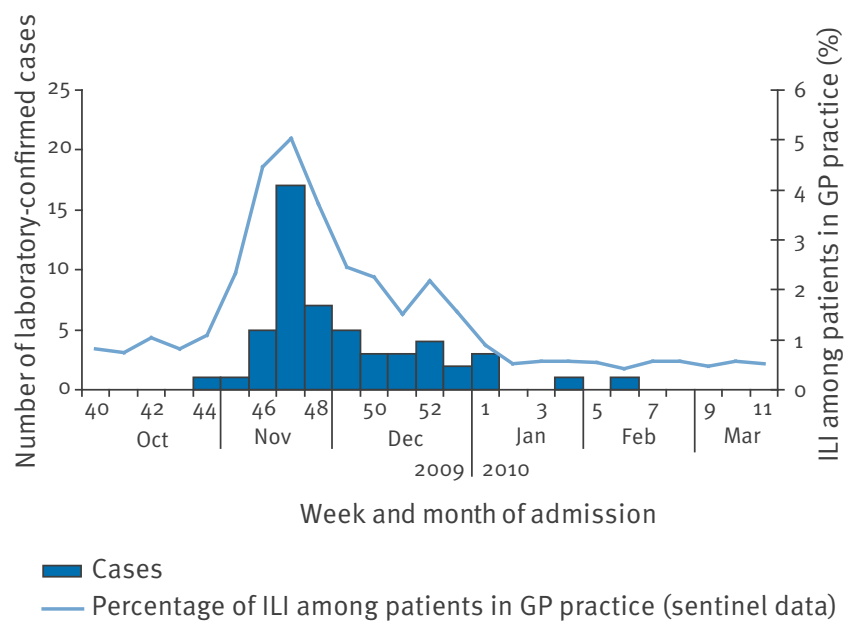

GP: general practioner; ILI: influenza-like illness.

\section{FIGURE 3}

Case-based data: incidence of laboratory-confirmed 2009 pandemic influenza $\mathrm{A}(\mathrm{H} 1 \mathrm{~N} 1)$ cases by sex and age group, Denmark, week 46 of 2009 to week 11 of 2010

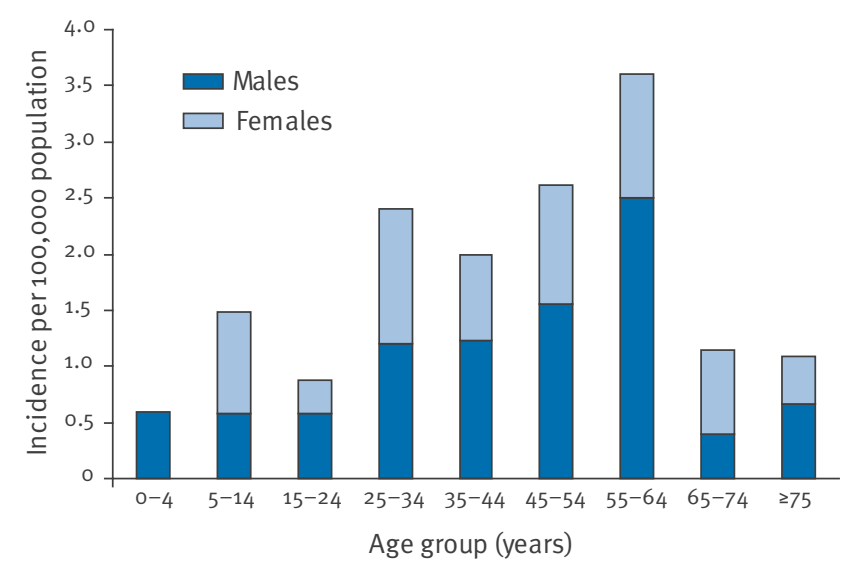


and 12 of 49 except for renal insufficiency, which was observed in fewer ( 3 of 49 ) cases. In addition, 14 of 47 of the cases had other underlying illnesses that were not further specified. One case was reported to have been pregnant when admitted to the ICU and one had recently given birth.
According to the vaccine registry 10 of the 53 cases had been vaccinated against pandemic influenza $A\left(\mathrm{H}_{1} \mathrm{~N}_{1}\right)$ : they had been vaccinated because of an underlying chronic illness. One of the 10 had been vaccinated twice, with an interval of a month between the vaccinations. The median time between vaccination and

\section{TABLE 1}

Case-based data: frequency of underlying conditions reported in cases with laboratory-confirmed 2009 pandemic influenza A(H1N1), Denmark, week 46 of 2009 to week 11 of $2010(n=52)$

\begin{tabular}{|c|c|c|c|c|c|c|c|}
\hline \multirow{2}{*}{ Underlying condition } & \multicolumn{2}{|l|}{ Age 0-14 years } & \multicolumn{2}{|l|}{ Age $\geq 15$ years } & \multicolumn{3}{|l|}{ Total } \\
\hline & Number of relevant cases & $\mathrm{n}$ & Number of relevant cases & $\mathrm{n}$ & Number of relevant cases & $\mathrm{n}$ & $\%$ \\
\hline None & 1 & 6 & 10 & 46 & 11 & 52 & 21.2 \\
\hline $\begin{array}{l}\text { Renal insufficiency (creatinine levels } \\
1.5 \text { times above normal) }\end{array}$ & 0 & 5 & 3 & 44 & 3 & 49 & 6.1 \\
\hline Cancer & 2 & 5 & 7 & 43 & 9 & 48 & 18.8 \\
\hline Immunocompromised condition & 3 & 5 & 6 & 42 & 9 & 47 & 19.1 \\
\hline Neurological illness & 2 & 5 & 7 & 42 & 9 & 47 & 19.1 \\
\hline Diabetes & 1 & 6 & 9 & 46 & 10 & 52 & 19.2 \\
\hline $\begin{array}{l}\text { Chronic lung disease, } \\
\text { including asthma }\end{array}$ & 1 & 5 & 10 & 44 & 11 & 49 & 22.4 \\
\hline Obesity (BMl>30) & NA & NA & 10 & 41 & 10 & 41 & 24.4 \\
\hline Cardiovascular disease & 1 & 5 & 11 & 44 & 12 & 49 & 24.5 \\
\hline Other underlying illness & 0 & 5 & 14 & 42 & 14 & 47 & 29.8 \\
\hline Pregnancy & NA & NA & 1 & 20 & 1 & 22 & 4.5 \\
\hline <42 days post-partum & NA & NA & 1 & 20 & 1 & 22 & $4 \cdot 5$ \\
\hline
\end{tabular}

BMI: body mass index; NA: not applicable.

\section{TABLE 2}

Case-based data: symptoms, treatment, interventions and outcome in cases with laboratory-confirmed 2009 pandemic influenza A(H1N1), Denmark, week 46 of 2009 to week 11 of 2010 (n=53)

\begin{tabular}{|c|c|c|c|}
\hline \multirow{2}{*}{ Description } & \multicolumn{3}{|c|}{ Total } \\
\hline & Number of relevant cases & $n$ & $\%$ \\
\hline \multicolumn{4}{|l|}{ Symptoms } \\
\hline Viral & 15 & 41 & 36.6 \\
\hline Bacterial & 5 & 41 & 12.2 \\
\hline Viral + bacterial & 21 & 41 & 51.2 \\
\hline Renal insufficiency (creatinine levels 1.5 times above normal) & 15 & 48 & 31.3 \\
\hline Septic shock & 19 & 50 & 38.0 \\
\hline \multicolumn{4}{|l|}{ Treatment and interventions } \\
\hline Antiviral treatment & 47 & 51 & 92.2 \\
\hline Oseltamivir alone & 27 & 47 & $57 \cdot 4$ \\
\hline Zanamivir alone & 1 & 47 & 2.1 \\
\hline No antiviral treatment & 4 & 51 & 7.8 \\
\hline Mechanical ventilation & 42 & 52 & 80.8 \\
\hline Invasive & 26 & 42 & 61.9 \\
\hline Non-invasive & 4 & 42 & $9 \cdot 5$ \\
\hline Invasive + non-invasive & 12 & 42 & 28.6 \\
\hline Haemodialysis & 10 & 50 & 20.0 \\
\hline Extracorporal membrane oxygenation & 6 & 53 & 11.3 \\
\hline \multicolumn{4}{|l|}{ Outcome } \\
\hline 30-day mortality & 17 & 53 & 32.1 \\
\hline
\end{tabular}


admission to an ICU was seven days (range: 3-35 days); seven cases were admitted to an ICU within 14 days of vaccination. Of the 41 patients reported to have at least one underlying medical condition in the case-based system, 32 were not vaccinated. The pregnant case who had been admitted to an ICU was not vaccinated.

\section{Clinical presentation, treatment,}

\section{interventions and outcome}

Table 2 shows the available data on clinical symptoms related to severe illness as well as treatment, interventions and outcome. The median interval between onset of symptoms and hospitalisation for 47 of the cases was three days (range: -78 to +33 ). Four of the 47 had already been hospitalised for 1, 5, 10 and 78 days when they developed pandemic influenza. When those four are excluded, the median time between symptom onset and hospital admission was four days. For these patients $(n=43)$, the median interval between hospital admission and ICU admission was one day (range: $\langle 1-21$ days,). The median time between onset of symptoms and the date of ICU admission was five days (range: $11-15$ days, with one outlier of 54 days, $n=43)$. The number of days in the ICU was calculated for 40 of these patients and ranged from less than one to 65 , with a median of 10 days.

A majority of patients ( 41 of 51 ) developed pneumonia and 19 of 50 had septic shock. Of 48 patients, 15 developed renal insufficiency, 12 of whom had no history of this condition. Ten patients developed both renal insufficiency and septic shock.

Of 51 patients, 47 were reported to have been treated with antiviral medication, mostly oseltamivir $(n=27)$ or a combination of oseltamivir and zanamivir $(n=19)$. The median interval between onset of symptoms and the start of any antiviral treatment was five days (range: -6 to +53 days, $n=42$ ). One person was already on antiviral treatment before symptom onset. The median interval between ICU admission and start of antiviral treatment was less than one day (range: -9 to +8 days, $n=47$ ). A total of 13 patients were on antiviral treatment before ICU admission. A total of 42 of 52 patients received mechanical ventilation: most of them received ventilation immediately when they were admitted to the ICU. The median time between admission and ventilation was less than one day (range: $\langle 1-4$ days, $n=42$ ). The median period of ventilation was 7.5 days (range: $<1-45$ days, $n=22$ ). Of 50 patients, 10 underwent haemodialysis and six of the 53 were treated with extracorporeal membrane oxygenation (ECMO).

The death rate was $32 \%$ ( 17 of the 53 cases). Three patients died more than 30 days after confirmation of their infection with pandemic influenza (34, 41 and 169 days after confirmation). As of 22 October 2010, the other 33 patients were alive. Of the 17 patients who died within 30 days 11 were male and six were female (Fisher's exact test $p=0.57$ ). Of the 17 cases whose deaths were related to the pandemic influenza,
13 had a pre-existing underlying medical condition. This was not associated with death (Fisher's exact test $p=1.0$ ). ECMO treatment was also not associated with a higher risk of death (three of six patients died after ECMO). Figure 4 shows the number of cases who died by age group. Of the 17 whose deaths were related to pandemic influenza, 12 were aged between 45 and 65 years.

\section{Discussion}

The aggregate data obtained through the surveillance system employed between week 46 of 2009 and week 11 of 2010 served as a tool to monitor the capacity in ICUs and to assist in planning for referral of severe cases as the epidemic progressed. Our results showed that the trend in incidence of pandemic influenza $A\left(\mathrm{H}_{1} \mathrm{~N}_{1}\right)$ infection was visible from the aggregate data even when only cases reported within the deadlines were considered. The aggregate data showed that the number of new cases reached its maximum a week later than the peak observed from the case-based surveillance and sentinel surveillance. This can be expected as the median period between onset of symptoms and ICU admission was five days.

The aggregate data enabled us to assess the number of patients with pandemic influenza in ICUs, but there are some uncertainties. We consider that the extent of the underestimation, due to inconsistent participation of some hospitals, is limited as we found that hospitals that had not reported usually had no cases. However, there might have been a slight overreporting of patients who had been transferred to another hospital. The choice of case definition, which included patients with an epidemiological link to a laboratory-confirmed patient, might have led to some false-positive cases. Due to the aggregate nature of the data, we cannot quantify this. All things considered, the extent of the uncertainties seems limited and we estimate that the number of reported cases $(n=93)$ closely approaches

\section{FIGURE 4}

Case-based data: laboratory-confirmed 2009 pandemic influenza $\mathrm{A}(\mathrm{H} 1 \mathrm{~N} 1)$ cases by outcome 30 days after initial laboratory confirmation and by age group, Denmark, week 46 of 2009 to week 11 of $2010(n=17)$

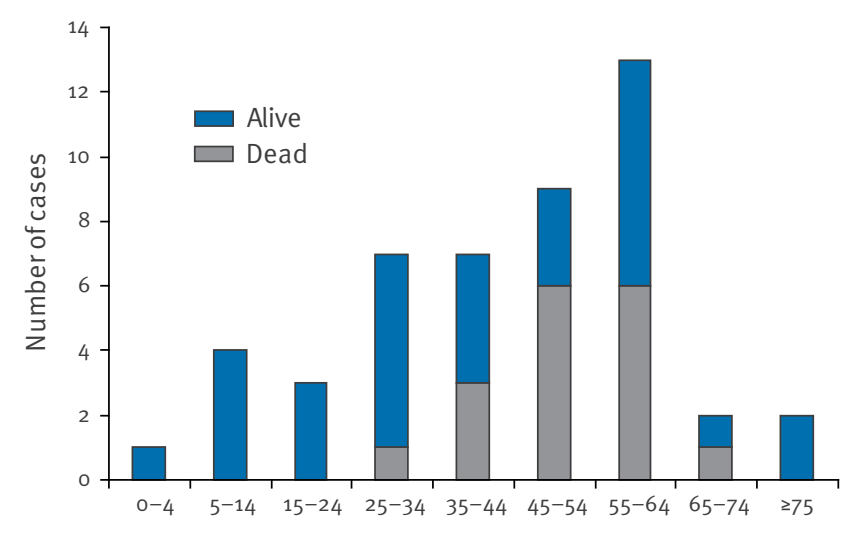

Age group (years) 
the actual number of patients with pandemic influenza in Danish ICUs. Therefore, the 53 confirmed cases used in the analysis of the case-based system can be assumed to represent approximately $57 \%$ (53 of 93) of the patients with pandemic influenza in Danish ICUs.

\section{Severity of the pandemic}

This surveillance system can assess certain aspects of the severity of the winter peak of the pandemic in Denmark: the number of severe cases in the general population, the death rate among severe cases and the specific groups that developed severe illness.

On the basis of the 93 cases reported in the aggregate data, the estimated incidence in Denmark (with a population of 5.5 million) was 1.7 per 100,000 population. This suggests that the overall impact of severe illness was not high at the population level and is in line with the incidence of ICU admissions observed in Australia and New Zealand during the 2009 winter peak [8]. In our study, the death rate was $32 \%$ (17 of 53 cases admitted to an ICU). These deaths occurred mainly in the age groups $44-54$ years and $55-64$ years. A cutoff point of 30 days after initial laboratory confirmation was chosen, to increase specificity, but it is possible that some of the deaths more than 30 days after confirmation were associated with pandemic influenza.

During seasonal influenza epidemics, children under two years of age and adults over 64 years are mostly affected, whereas the 2009 pandemic typically affected young adults [3-5]. The World Health Organization stated that people older than 65 years were the least likely to be infected with pandemic influenza, but if infected they would be at high risk of developing serious complications [9]. In Denmark, children aged 5-14 years contributed heavily to the number of patients admitted to hospitals [10], which was less prominent in the ICU admissions. The median age of 47 years of the cases in our study is within the range described in other studies of ICU patients with pandemic influenza [11-16]. While healthy adults generally do not suffer from severe illness when infected with seasonal influenza, the pandemic showed a different picture worldwide [3-5]. Our case-based data also showed a relatively high number of severe cases among previously healthy individuals.

The pandemic vaccination campaign started in week 45 of 2009 in Denmark. The strategy - to vaccinate all individuals with risk factors independent of age - was in line with the wide range in age distribution seen among patients with pandemic influenza in ICUs. It is important to note that the majority of the reported ICU cases with an underlying disease was not vaccinated. For those ICU patients who were vaccinated the vaccine probably came too late. However, vaccine effectiveness studies are needed to draw conclusions on these issues.
Severity of disease

The median period of five days between onset of symptoms of pandemic influenza and ICU admission was consistent with observations in other studies in Argentina (median of six days) and in Australia, New Zealand and Canada (median of four days) [11-13]. This interval will be influenced by access to healthcare and the perception of severity of symptoms by patients and physicians.

Severe respiratory failure occurred in 42 of 52 cases and for most of them, mechanical ventilation was started the same day they were admitted to the ICU. Also in other ways, the clinical presentation of pandemic influenza patients in Danish ICUs was severe, with 10 of 48 cases developing both renal insufficiency and septic shock, and several cases developing either renal insufficiency or septic shock. Davies et al. predicted that Europe had to prepare for an estimated 2.6 persons per million inhabitants needing ECMO treatment as a result of pandemic influenza [12]. Since ECMO treatment was only performed in one hospital in Denmark during the pandemic, we could verify that the six cases reported in our case-based surveillance to have received ECMO were in fact all pandemic influenza cases in Denmark who received ECMO during the surveillance period. This number is of the order of magnitude Davies et al. predicted.

\section{Impact of the pandemic on}

\section{Danish intensive care units}

The aggregate data showed that the burden on the ICUs was limited, at a national level. However, for hospitals that had pandemic influenza cases the ICU bed capacity used for these patients was substantial. Similar findings on ICU bed capacity were reported from Australia and New Zealand during the 2009 winter peak [8]. Our case-based data showed that the vast majority of cases needed ventilation and a high number of cases presented with complications, requiring treatment such as haemodialysis and ECMO. This required a high level of care and led to extra pressure on ICU facilities and staff. Due to the absence of baseline data it is, however, not possible to compare this to the situation in ICUs during seasonal influenza epidemics.

The combination of aggregate and case-based data proved to be a useful tool to assess the situation in ICUs during the 2009 pandemic. Since both epidemic curves followed the same trend as the data from sentinel surveillance and on-call monitoring, the sentinel and on-call systems can be used to decide when to put the ICU surveillance in place during the next winter season. The ICU surveillance system could also be used during a seasonal epidemic in order to learn more about the baseline situation for seasonal influenza.

Acknowledgements

We wish to thank all contact persons in the ICUs for their efforts to provide us with the information and the clinical 
microbiology laboratories in Denmark for sending the samples to the Statens Serum Institut. We would also like to thank Eskild Petersen (Department of Infectious Diseases, Aarhus University Hospital, Skejby, Aarhus, Denmark), Per Bo Larsen (Unit for Acute Medicine and Health Preparedness, Capital Region, Hillerød, Denmark), Jens Lundgren (University of Copenhagen and Rigshospitalet, Copenhagen, Denmark), Michael Galle, Kenn Schultz Nielsen, Jakob Holck, Katarina Widgren, Steffen Offersen Glismann, Anne Mazick and Tyra Krause (Department of Epidemiology, Statens Serum Institut, Copenhagen, Denmark), Lars Peter Nielsen and Betina Skovgaard Andresen (Virology laboratory, Statens Serum Institut, Copenhagen, Denmark) and the EPIET coordinators.

\section{References}

1. Statens Serum Institut. Influenza-Nyt - uge 5, 2010. [InfluenzaNews - week 5, 2010] Feb 2010. Danish. Available from: http:// www.ssi.dk/Aktuelt/Nyhedsbreve/INFLUENZA-NYT/2009-2010/ INFLUENZA-NYT\%20-\%20uge\%205\%20-\%202010.aspx

2. Harder KM, Andersen PH, Bæhr I, Nielsen LP, Ethelberg S, Glismann S, et al. Electronic real-time surveillance for influenza-like illness: experience from the 2009 influenza $A\left(\mathrm{H}_{1} \mathrm{~N}_{1}\right)$ pandemic in Denmark. Euro Surveill. Forthcoming 2011.

3. ECDC working group on influenza $A\left(\mathrm{H}_{1} \mathrm{~N}_{1}\right) v$. Preliminary analysis of influenza $A\left(\mathrm{H}_{1} \mathrm{~N}_{1}\right) v$ individual and aggregated case reports from EU and EFTA countries. Euro Surveill. 2009;14(23):pii=19238. Available from: http://www. eurosurveillance.org/ViewArticle.aspx?Articleld=19238.

4. Health Protection Agency and Health Protection Scotland new influenza $A\left(\mathrm{H}_{1} \mathrm{~N}_{1}\right)$ investigation teams. Epidemiology of new influenza $A\left(\mathrm{H}_{1} \mathrm{~N}_{1}\right)$ in the United Kingdom, April - May 2009. Euro Surveill. 2009;14(19):pii=19213. Available from: http:// www.eurosurveillance.org/ViewArticle.aspx?Articleld=19213

5. Fraser C, Donnelly CA, Cauchemez S, Hanage WP, Van Kerkhove MD, Hollingsworth TD, et al. Pandemic potential of a strain of influenza $A\left(\mathrm{H}_{1} \mathrm{~N}_{1}\right)$ : early findings. Science. 2009;324(5934):1557-61.

6. Sundhedsstyrelsen. Beredskab for pandemisk influenza, 2006. [Preparedness for pandemic influenza 2006]. Apr 2006. Danish. Available from: http://www.sst.dk/publ/Publ2006/ CFF/Pandemibered/pandemiplan2006.pdf

7. Pedersen CB, Gøtzsche H, Møller JO, Mortensen PB. The Danish Civil Registration System. A cohort of eight million persons. Dan Med Bull. 2006;53(4):441-9.

8. ANZIC Influenza Investigators, Webb SA, Pettilä V, Seppelt I, Bellomo R, Bailey M, et al. Critical care services and 2009 $\mathrm{H}_{1} \mathrm{~N}_{1}$ influenza in Australia and New Zealand. N Engl J Med. 2009;361(20):1925-34.

9. World Health Organization (WHO). Who is more at risk of severe illness? What about other risks? 24 Feb 2010. Available from: http://www.who.int/csr/disease/swineflu/frequently_ asked_questions/risk/en/index.html

10. Widgren K, Nielsen J, Mølbak K. Registry-based surveillance of influenza-associated hospitalisations during the 2009 influenza pandemic in Denmark: the hidden burden on the young. PLoS One. 2010;5(11):e13939.

11. Kumar A, Zarychanski R, Pinto R, Cook DJ, Marshall J, Lacroix J, et al. Critically ill patients with 2009 influenza $A\left(\mathrm{H}_{1} \mathrm{~N}_{1}\right)$ infection in Canada. JAMA. 2009;302(17):1872-9.

12. Australia and New Zealand Extracorporeal Membrane Oxygenation (ANZ ECMO) Influenza Investigators, Davies A, Jones D, Bailey M, Beca J, Bellomo R, et al. Extracorporeal membrane oxygenation for 2009 influenza $A\left(\mathrm{H}_{1} \mathrm{~N}_{1}\right)$ acute respiratory distress syndrome. JAMA. 2009;302(17):1888-95.

13. Estenssoro E, Ríos FG, Apezteguía C, Reina R, Neira J, Ceraso $\mathrm{DH}$, et al. Pandemic 2009 influenza $A\left(\mathrm{H}_{1} \mathrm{~N}_{1}\right)$ in Argentina: a study of 337 patients on mechanical ventilation. Am J Respir Crit Care Med. 2010;182(1):41-8.

14. Louie JK, Acosta M, Winter K, Jean C, Gavali S, Schechter R, et al. Factors associated with death or hospitalization due to pandemic 2009 influenza $A\left(\mathrm{H}_{1} \mathrm{~N}_{1}\right)$ infection in California. JAMA. 2009;302(17):1896-902.

15. Koegelenberg CF, Irusen EM, Cooper R, Diacon AH, Taljaard JJ, Mowlana A, et al. High mortality from respiratory failure secondary to swine-origin influenza $A\left(\mathrm{H}_{1} \mathrm{~N}_{1}\right)$ in South Africa. QJM. 2010;103(5):319-25.

16. Domínguez-Cherit G, Lapinsky SE, Macias AE, Pinto R, Espinosa-Perez $L$, de la Torre A, et al. Critically Ill patients with 2009 influenza $A\left(\mathrm{H}_{1} \mathrm{~N}_{1}\right)$ in Mexico. JAMA. 2009;302(17):1880-7. 